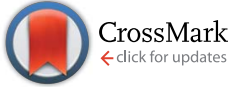

Cite this: RSC Adv., 2017, 7, 9975

\title{
CASSCF and CASMP2 study on the photoisomerization mechanisms of [tris(trialkylsilyl)silyl]cyclotetrasilene and related cyclobutene molecules $\dagger$
}

\begin{abstract}
Ming-Der Su*ab
The mechanisms for photochemical isomerization reactions are studied theoretically using the model systems, cyclotetrasilene and cyclobutene, both of which feature one four-membered-ring and one double bond, using the CAS(6,6)/6-311G(d) and CAS-MP2-(6,6)/6-311++G(3df,3pd) methods. The mechanisms, which are governed by the conical intersection concept, play a crucial role in these photorearrangement reactions, and explain the reaction pathways. The model computations demonstrate that the preferred reaction path for the photoisomerization reactions for both cyclotetrasilene and cyclobutene is as follows: four-membered-ring reactant $\rightarrow$ Franck-Condon region $\rightarrow$ conical intersection $\rightarrow$ butterfly-like photoproduct. These results allow a number of predictions to be made.
\end{abstract}

Received 12th January 2017 Accepted 30th January 2017

DOI: $10.1039 / \mathrm{c} 7 \mathrm{ra00506g}$

rsc.li/rsc-advances

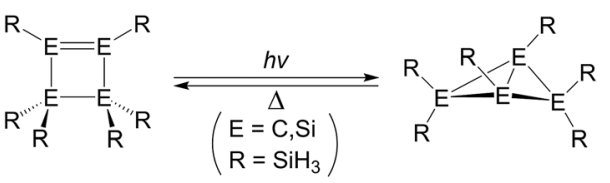

Scheme 2 properties. ${ }^{1,2}$ However, only very few photochemical reactions that involve the disilene systems have been studied experimentally. ${ }^{3}$

About 15 years ago, Kira and co-workers reported a distinctive photochemical reaction that rearranges hexakis(tert-butyldimethylsilyl)cyclotetrasilene (I) to its corresponding isomer, hexakis(tert-butyldimethylsilyl)-bicyclo[1,1,0]tetrasilane (II), as shown in Scheme $1 .{ }^{4}$ After heating, (II) is isomerized back to the initial reactant (I). However, to the best of the author's knowledge, there has been no theoretical study of the mechanism for this photo-isomerization reaction for cyclotetrasilene (I) ${ }^{4 c}$ This may be because the computational methods for studying

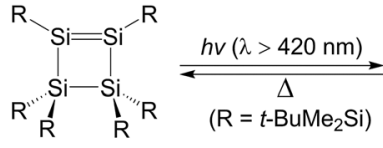

I

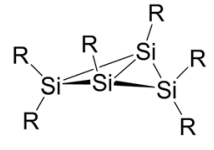

II

Scheme 1

${ }^{a}$ Department of Applied Chemistry, National Chiayi University, Chiayi 60004, Taiwan. E-mail: midesu@mail.ncyu.edu.tw

${ }^{b}$ Department of Medicinal and Applied Chemistry, Kaohsiung Medical University, Kaohsiung 80708, Taiwan

$\dagger$ Electronic supplementary information (ESI) available. See DOI: $10.1039 / \mathrm{c} 7 \mathrm{ra00506g}$

photochemistry were not so mature at that time. The author

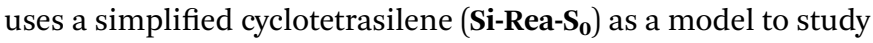
its photochemical mechanism for the isomerization reaction to bicyclo[1,1,0]tetrasilane (Si-Pro-S $\mathbf{S}_{\mathbf{0}}$ ) using modern theoretical methods, i.e., CASSCF (the complete-active-space SCF) ${ }^{5}$ and the multi-reference Møller-Plesset (CAS-MP2) algorithm. ${ }^{6}$ For the purposes of comparison, a similar mechanistic study is also made of a four-carbon system, C-Rea- $\mathbf{S}_{\mathbf{0}}$, as represented in Scheme $2 .^{7}$

Conical intersection (CI) points ${ }^{8}$ can occur on the potential surfaces, from which the excited state decays to the ground state surface at the same multiplicity (usually from singlet to singlet). Therefore, the CI mechanism is used in this work to interpret the reaction trajectories for the photochemistry of both Si-Pro$\mathbf{S}_{\mathbf{0}}$ and $\mathbf{C}-\mathbf{P r o}-\mathbf{S}_{\mathbf{0}}$ molecules. This paper shows that the CIs ${ }^{\mathbf{8}}$ play a decisive role in the photo-rearrangement reactions for cyclotetrasilene and cyclobutene molecules, which have a fourmembered-ring.

\section{Theoretical methods}

The CASSCF computations are executed using the MCSCF $^{9}$ and CAS-MP2 (ref. 9) programs in GAUSSIAN 09. ${ }^{10}$ The active space 
that is used to describe the photo-rearrangements for the fourmembered-ring molecules (Scheme 2) that are studied in this work include six electrons in six orbitals, i.e., $1 \pi(\mathrm{E}=\mathrm{E})+$ $1 \pi^{*}(\mathrm{E}=\mathrm{E})+2 \sigma(\mathrm{E}-\mathrm{E})+2 \sigma^{*}(\mathrm{E}-\mathrm{E})$ orbitals. The state-averaged $\operatorname{CASSCF}(6,6)$ method was adopted to verify geometry on the intersection space (i.e., root $=2$ ). Moreover, the excited state gradient was obtained by using the CSASSCF method. The harmonic frequencies are computed analytically at the $\operatorname{CAS}(6,6) /$ 6-311G(d) level of theory and are used to characterize either minima or transition states. Unless otherwise stated, the relative energies that are shown in this study are those that are ascertained at the CAS-MP2(6,6)/6-311++G(3df,3pd) level, using the CAS(6,6)/6-311G(d) geometry. These are designated CASMP2 and CASSCF, respectively. The Cartesian coordinates and the energies that are computed for the various points are available as ESI. $\dagger$

\section{General considerations}

According to the available experimental results, ${ }^{4}$ there is no indication that a triplet state participates in these photoisomerization reactions. The CAS-MP2 computational results also show that the relative energies $\left(\mathrm{kcal} \mathrm{mol}^{-1}\right)$ for the FranckCondon (FC) regions are as follows: Si-Rea-S $\mathbf{S}_{\mathbf{0}}(0.0)<$ Si-FC-S $_{\mathbf{1}}$ $(68.8)<$ Si-FC-T $\mathbf{1}$ (93.6) and C-Rea-S $\mathbf{S}_{\mathbf{0}}(0.0)<$ C-FC-S $_{\mathbf{1}}(116.8)<$ CFC-T $_{1}$ (121.8). ${ }^{11}$ From these theoretical results, it is concluded that triplet states do not play any role in the photochemical reactions for these cyclic disilene molecular systems, so this study concentrates on singlet surfaces for both Si-Rea-S $\mathbf{S}_{\mathbf{0}}$ and CRea-S $S_{0}$ reactants. ${ }^{12}$

In order to determine possible excited-state reaction paths that lead to CIs, a molecular orbital representation of the relationship between the likely reaction pathway and intersection is schematically illustrated in Scheme $3(\mathrm{E}=\mathrm{C}$ and $\mathrm{Si}),{ }^{13}$ which is a foundation for these theoretical studies. Upon irradiation with light, a singlet four-membered-ring molecule (1) is moved up to a singlet excited species (2), succeeding an intramolecular double bond $(\mathrm{E}=\mathrm{E})$ rotation. Then, as shown in (3), this excited molecule 1 can be considered as a composition of four kinds of carbenes $(>\mathrm{E})$, existing four spin-up $\sigma$ orbitals and four spin-
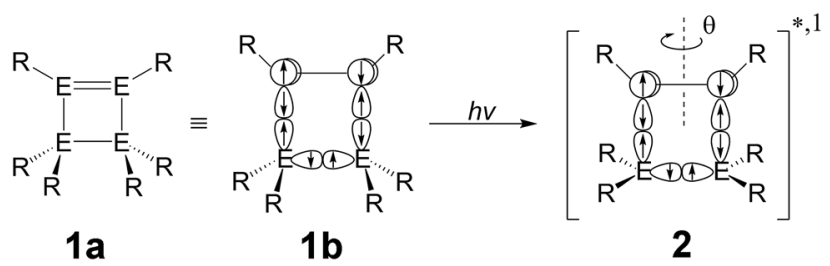

2

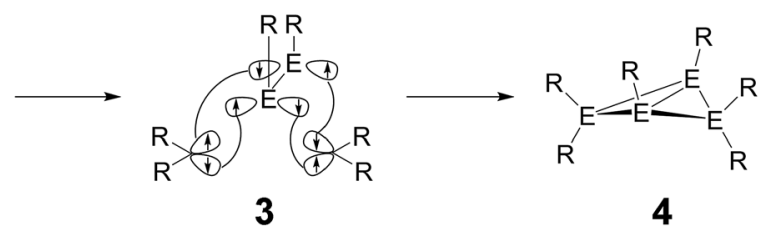

Scheme 3
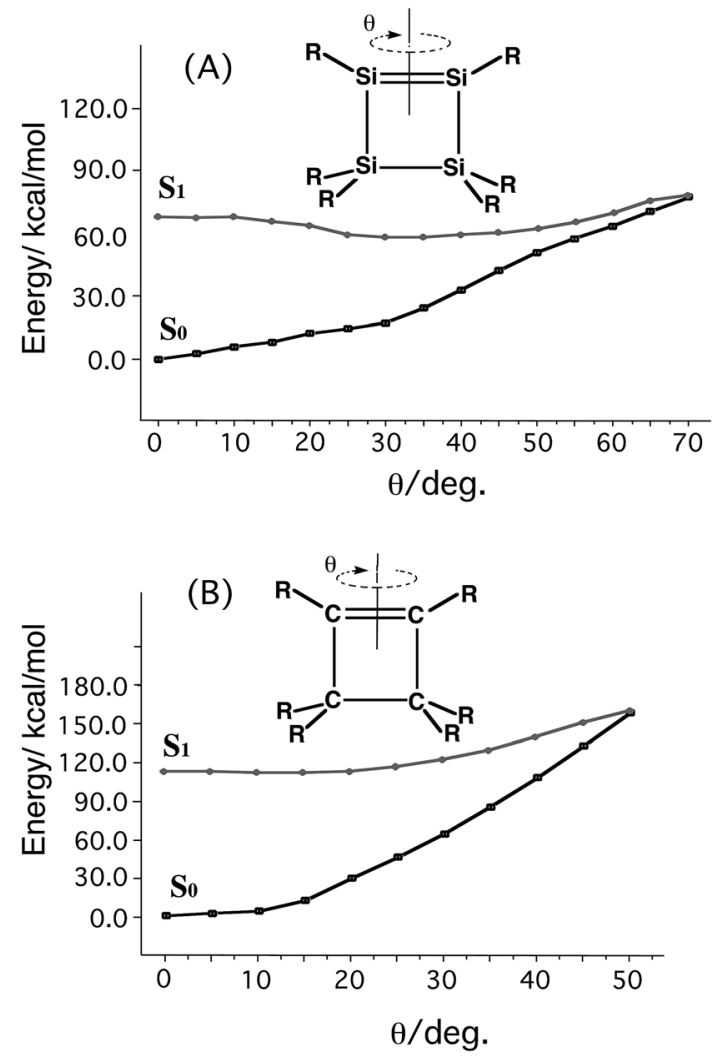

Fig. 1 The scans for finding a $\mathrm{Cl}$ pathway using a $\mathrm{E}=\mathrm{E}(\mathrm{E}=\mathrm{C}$ and $\mathrm{Si})$ rotation in the reactants ((A) Si-Rea- $\mathrm{S}_{0}$ and (B) C-Rea- $\mathrm{S}_{0} ; \mathrm{R}=\mathrm{SiH}_{3}$ ) for the angle, $\theta$, optimized for the $S_{0}$ and $S_{1}$ states at the CAS $(6,6) / 6$ $311 \mathrm{G}(\mathrm{d})$ level of theory. See the text and ESI. $\dagger$

down $\sigma$ orbitals. ${ }^{13}$ Consequently, through the conical intersection, a two-three-membered-ring photoproduct (4) can be obtained.

Using the molecular orbital analysis that is given in Scheme 3 , since the narrow energy gap between $S_{0}$ and $S_{1}$ energy surfaces strongly implies the existence of a CI point, Si-Rea-S $\mathbf{S}_{\mathbf{0}}$ and $\mathbf{C}-\mathbf{R e a - S _ { \mathbf { 0 } }}$ are used as examples to determine whether there is a low-lying $S_{1} / S_{0}$ surface crossing. Fig. 1 shows the potential energy profiles for the rotational angle, $\theta$, as shown in Scheme 3. ${ }^{14}$ Fig. 1(A) and (B) show that the reaction pathways all terminate at a very similar $S_{1}-S_{0}$ energy gap at around $75^{\circ}$ and $60^{\circ}$, respectively. These energy profile results suggest that a CI point occurs in the intramolecular rotation for the fourmembered-ring geometries. In the next section, these findings are used to explain the mechanisms for the photochemical

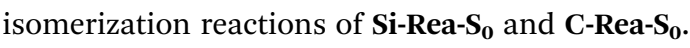

\section{Results and discussion}

The discussion in Section III shows that there should exist a reaction route for photochemical isomerization reactions on the singlet excited energy surfaces of Si-Rea-S $\mathbf{S}_{\mathbf{0}}$ that contains a conical intersection point. Fig. 2 shows the energies for the key points relative to the energy of the reactant, Si-Rea-S. The optimized geometries for these important points on the 


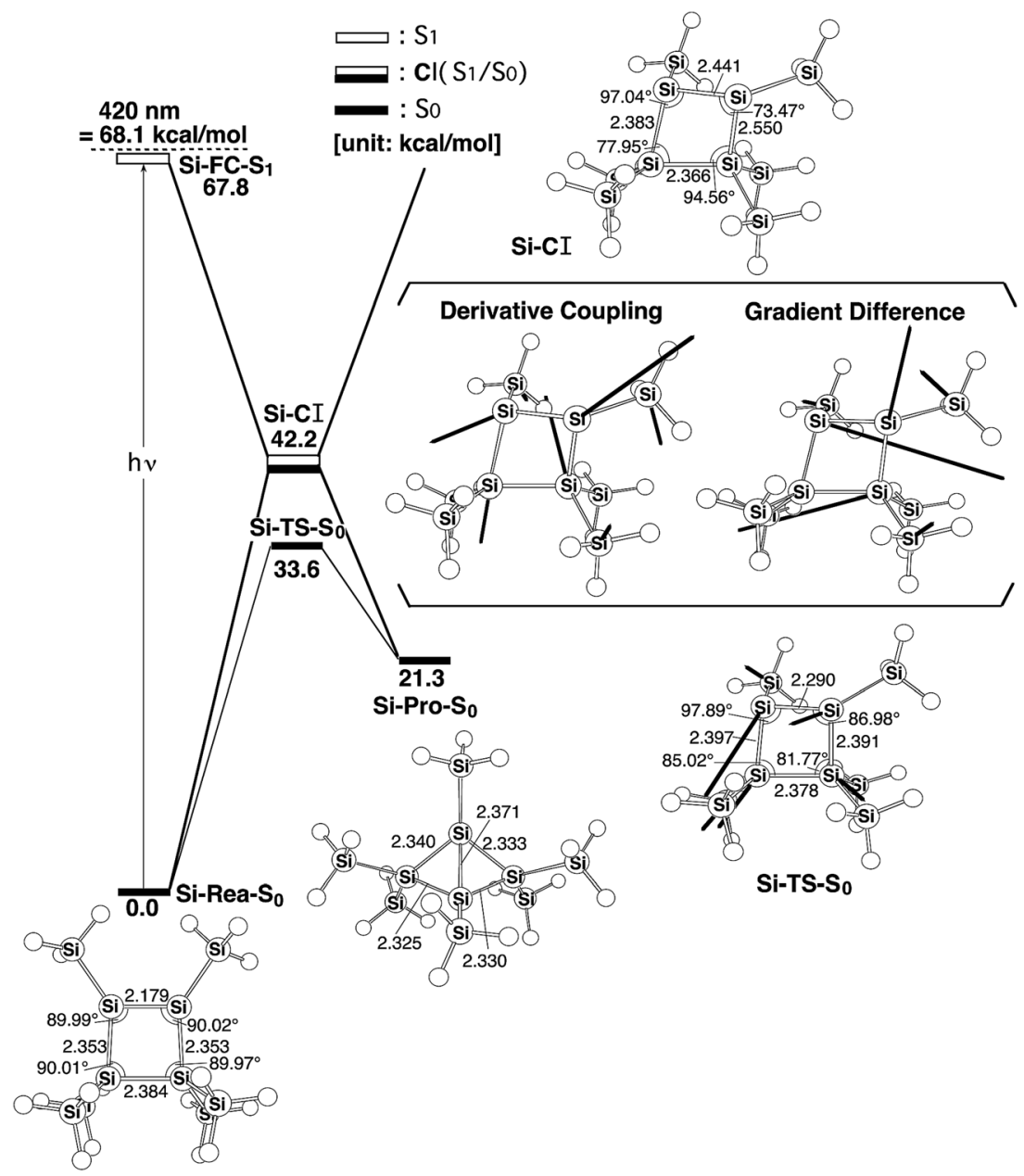

Fig. 2 The energy surfaces for the photochemical isomerization reaction for Si-Rea- $\mathrm{S}_{0}$. The abbreviations, FC, TS and Cl, denote FranckCondon, transition state and conical intersection, respectively. The relative energies are obtained at the CAS-MP2-(6,6)/6-311++G(df,pd)// CAS $(6,6) / 6-311 \mathrm{G}(\mathrm{d})$ level of theory. The geometrical parameters for the CASSCF-optimized structures for the stationary points are also shown. Hydrogen atoms are omitted for clarity. The bold arrows show the main atomic motions in the Si-TS- $\mathrm{S}_{0}$ eigenvector. The derivative coupling and the gradient difference vectors - those which increase the degeneracy - are computed using CASSCF at the conical intersection, Si-CI. For more information see the text.

mechanistic pathways in Fig. 2 are also shown in the same figure.

A comparison with the CASSCF-optimized geometry for the silicon reactant (Si-Rea-S $\mathbf{S}_{\mathbf{0}}$ ), as shown in Fig. 2, shows that the theoretical values for the bond lengths and the bond angles in these four-membered-ring species are in good agreement with the experimental values. ${ }^{4}$ The bond lengths and the bond angles are within $0.07 \AA$ and $5.9^{\circ}$, respectively. Therefore, CASSCF and CAS-MP2 computations should be satisfactory for a theoretical study of the photochemical and thermal reactions.

Initially, cyclotetrasilene (Si-Rea-S $\mathbf{S}_{\mathbf{0}}$ ) with a four-siliconmembered-ring is irradiated by visible light and is promoted to the Franck-Condon region at the first excited singlet state (Si-

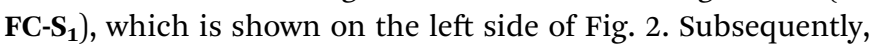
this species relaxes to the conical intersection point (Si-CI), from which it can go to either the final photoproduct (Si-Pro-S $\mathbf{S}_{\mathbf{0}}$ ) or the initial reactant $\left(\mathbf{S i}-\mathbf{R e a}-\mathbf{S}_{\mathbf{0}}\right)$. These CAS-MP2 computations show that the relative energies for Si-FC-S $_{\mathbf{1}}, \mathbf{S i - C I}$, and Si-Pro-S $\mathbf{S}_{\mathbf{0}}$ with respect to Si-Rea-S $\mathbf{S}_{\mathbf{0}}$ are 68, 42, and $21 \mathrm{kcal} \mathrm{mol}^{-1}$, respectively. The theoretical evidence strongly suggests that when cyclotetrasilene (Si-Rea-S $\mathbf{S}_{\mathbf{0}}$ ) is irradiated by visible light, it easily undergoes a $\mathrm{Si}=\mathrm{Si}$ double bond rotation to produce either the reactant $\left(\mathbf{S i - R e a - S _ { 0 } )}\right.$ or the corresponding bicyclo[1,1,0]tetrasilane (Si-Pro-S $\left.\mathbf{S}_{\mathbf{0}}\right)$. The photochemical isomerization mechanism for Si-Rea-S $\mathbf{S}_{\mathbf{0}}$ that is shown in Fig. 2 is represented as follows:

$$
\begin{gathered}
\text { Cyclotetrasilene (photo): } \\
\text { Si-Rea-Si-Pro-S } \mathbf{S}_{\mathbf{0}}+h \nu \rightarrow \text { Si-FC-S }_{\mathbf{1}} \rightarrow \text { Si-CI } \rightarrow
\end{gathered}
$$

The thermal (dark) reaction on the ground singlet state energy surface that is shown in Fig. 2 is also studied. Fig. 2 shows that the $\mathbf{S i - T S}-\mathbf{S}_{\mathbf{0}}$ point combines $\mathbf{S i - R e a - S _ { \mathbf { 0 } }}$ and $\mathbf{S i - P r o - S _ { \mathbf { 0 } }}$ on the ground singlet surface and that its energy lies $15 \mathrm{kcal}$ $\mathrm{mol}^{-1}$ below the energy of the Si-CI point. It is worthy of note that the CAS-MP2 calculations give barrier heights for Si-Rea-S $\rightarrow$ Si-TS-S $\mathbf{S}_{\mathbf{0}}$ and Si-Pro-S $\mathbf{S}_{\mathbf{0}} \rightarrow$ Si-TS-S $\mathbf{S}_{\mathbf{0}}$ that are approximately 34 
and $12 \mathrm{kcal} \mathrm{mol}^{-1}$, respectively. This theoretical evidence points to the fact that cyclotetrasilene $\left(\mathbf{S i}-\mathbf{R e a}-\mathbf{S}_{\mathbf{0}}\right)$ is thermodynamically more stable than bicyclo[1,1,0]tetrasilane $\left(\mathbf{S i}-\mathbf{P r o}-\mathbf{S}_{\mathbf{0}}\right)$ and producing the latter from the former using the thermal reaction would be difficult, which is in good agreement with the experimental results. ${ }^{4}$ Fig. 2 also predicts that the thermal reversion from Si-Pro- $\mathbf{S}_{\mathbf{0}}$ to $\mathbf{S i - R e a - S _ { 0 }}$ is relatively easily accomplished, since the energy that is required to surmount the reverse barrier $\left(12 \mathrm{kcal} \mathrm{mol}^{-1}\right.$ ) is less than that which is required to surmount forward barrier $\left(34 \mathrm{kcal} \mathrm{mol}^{-1}\right)$. This theoretical prediction is again in good agreement with the available experimental results. ${ }^{4}$

The photochemical conversion of $\mathbf{C}-\mathbf{R e a}-\mathbf{S}_{\mathbf{0}}$ to $\mathbf{C}-\mathbf{P r o}-\mathbf{S}_{\mathbf{0}}$ and its thermal reversion are studied. The computational results are all listed in Fig. 3. Similarly to the model for cyclotetrasilene
(Si-Rea-S $\mathbf{S}_{\mathbf{0}}$ ), the singlet photo-rearrangement reaction for C-Rea$\mathbf{S}_{\mathbf{0}}$ that is studied in this work follows similar reaction paths to those that are shown in Fig. 2. The CAS-MP2 computations suggest that the photo-isomerization reaction for C-Rea-S proceeds as follows:
Cyclobutene (photo): C-Rea-S $\mathbf{0}_{\mathbf{0}}+h \nu \rightarrow \mathbf{C}-\mathbf{F C}-\mathbf{S}_{\mathbf{1}} \rightarrow \mathbf{C}-\mathbf{C I} \rightarrow \mathbf{C}-$ Pro-S

However, the CAS-MP2 results demonstrate that much higher energy (117 $\mathrm{kcal} \mathrm{mol}^{-1}$ ) of $\mathbf{C}-\mathbf{F C}-\mathbf{S}_{\mathbf{1}}$ than that of the irradiating light $\left(420 \mathrm{~nm}=68 \mathrm{kcal} \mathrm{mol}^{-1}\right)$ makes this photorearrangement reaction energetically unfeasible, ${ }^{15}$ so the energy of photo-irradiation is insufficient to generate the butterfly-like product, $\mathbf{C}-\mathbf{P r o}-\mathbf{S}_{\mathbf{0}}$ via radiation-less decay (i.e., the

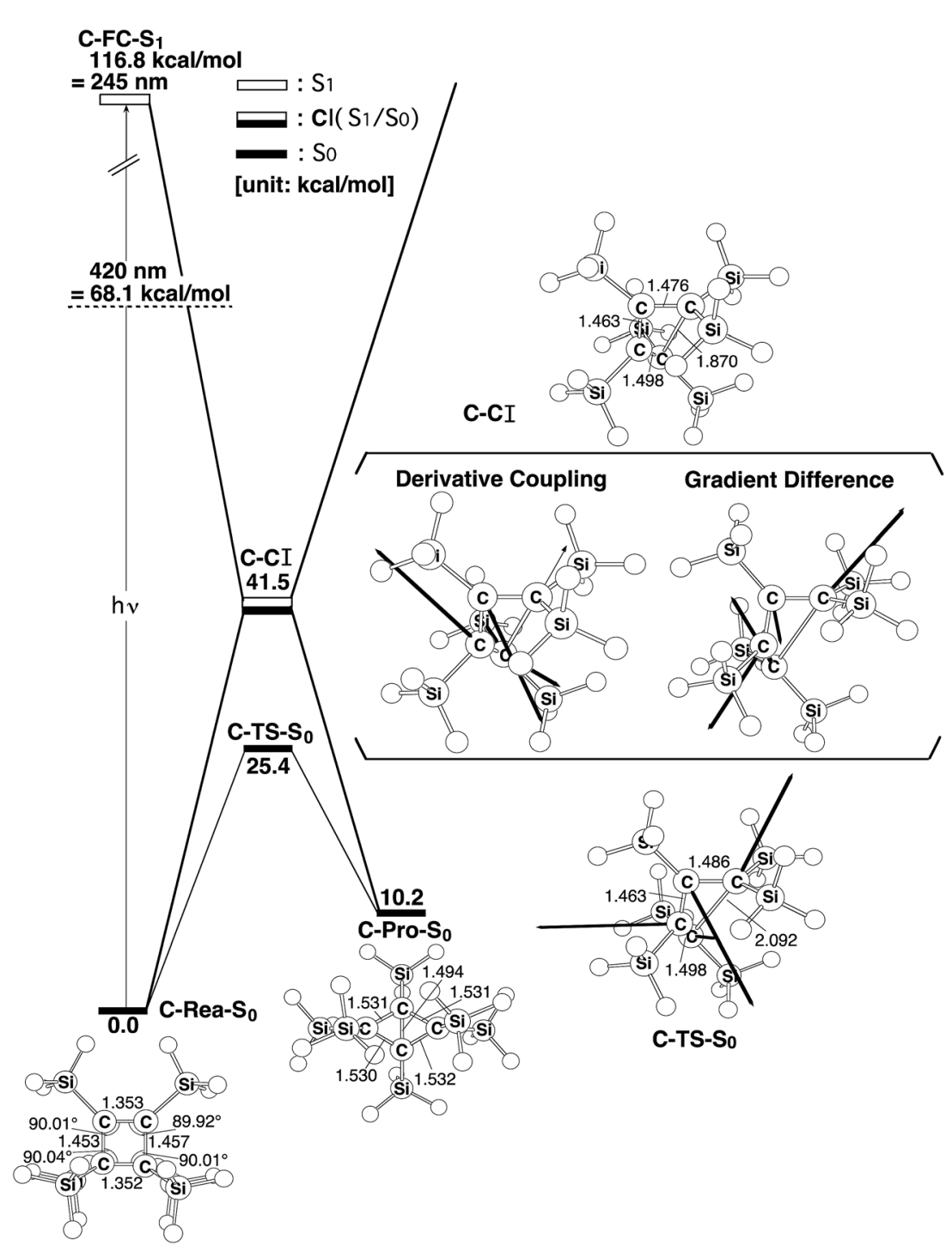

Fig. 3 The energy surfaces for the photochemical isomerization reaction for C-Rea- $\mathrm{S}_{0}$. The abbreviations, FC, TS and $\mathrm{Cl}$, denote FranckCondon, transition state and conical intersection, respectively. The relative energies are obtained at the CAS-MP2-(6,6)/6-311++G(df,pd)// CAS $(6,6) / 6-311 \mathrm{G}(\mathrm{d})$ level of theory. The geometrical parameters for the CASSCF-optimized structures for the stationary points are also shown. Hydrogen atoms are omitted for clarity. The bold arrows show the main atomic motions in the C-TS-So eigenvector. The derivative coupling and the gradient difference vectors - those that increase the degeneracy - are computed using CASSCF at the conical intersection, $\mathrm{C}$-Cl. For more information, see the text. 
CI channel). Consequently, no photo-isomerization reaction should be observed in these cyclobutene systems for irradiation with visible light. However, the thermal reversion (C-Pro-S $\mathbf{S}_{\mathbf{0}} \rightarrow$ C-Rea-S $\mathbf{S}_{\mathbf{0}}$ ) is predicted to be easily achieved, since the energy that is required to surmount the reverse barrier $\left(\mathbf{C}-\mathbf{P r o}-\mathbf{S}_{\mathbf{0}} \rightarrow \mathbf{C}\right.$ TS- $\mathbf{S}_{\mathbf{0}}$ ) is calculated to be $10 \mathrm{kcal} \mathrm{mol}^{-1}$ less than that which is required to surmount the forward barrier (C-Rea-S $\mathbf{S}_{\mathbf{0}} \rightarrow \mathbf{C}$-TS-S $\mathbf{S}_{\mathbf{0}}$ ), using the CAS-MP2 calculations. To the best of the author's knowledge, no experimental reports of the photochemical reactions of cyclobutenes have been published so the CAS-MP2 computational results that are shown in Fig. 3 remain a prediction.

\section{Conclusion}

The photochemical mechanisms for the isomerization reactions for $\mathbf{S i - R e a - S _ { 0 }}$ and $\mathbf{C}-\mathbf{R e a}-\mathbf{S}_{\mathbf{0}}$, which have a skeleton that has one four-membered-ring and one double bond, are studied using the CASSCF and CAS-MP2 levels of theory. This is the first theoretical study of the reaction trajectory and the first theoretical estimation of the activation energy and the reaction enthalpy for both the singlet excited state and the singlet ground state. This study interprets the photochemical behavior of these four-membered-ring species that contain group 14 elements using a simple conical intersection mechanism. The available experimental findings for cyclotetrasilene are verified ${ }^{4}$ and the photochemical isomerization reactions for analogous cyclobutene systems are predicted.

\section{Acknowledgements}

The author is grateful to the National Center for HighPerformance Computing of Taiwan for generous amounts of computing time, and the Ministry of Science and Technology of Taiwan for the financial support. In particular, the author also wishes to thank Professor Michael A. Robb, Dr S. Wilsey, Dr Michael J. Bearpark, (University of London, UK) and Professor Massimo Olivucci (Universita degli Studi di Siena, Italy), for their encouragement and support during his stay in London. Special thanks are also due to reviewers 1 and 2 for very help suggestions and comments.

\section{References}

1 R. West, M. J. Fink and J. Michl, Science, 1981, 214, 1343.

2 For reviews on disilenes, see: (a) R. West, Pure Appl. Chem., 1984, 56, 163; (b) G. Raabe and J. Michl, Chem. Rev., 1985, 85, 419; (c) A. H. Cowley and N. C. Norman, Prog. Inorg. Chem., 1986, 34, 1; (d) R. West, Angew. Chem., Int. Ed. Engl., 1987, 26, 1201; (e) G. Raabe and J. Michl, in The Chemistry of Organic Silicon Compound, ed. S. Patai and Z. Rappoport, Wiley, New York, 1989, part 2, ch. 17; (f) T. Tsumuraya, S. A. Batcheller and S. Masamune, Angew. Chem., Int. Ed. Engl., 1991, 30, 902; (g) R. S. Grev, Adv. Organomet. Chem., 1991, 33, 125; (h) M. Weidenbruch, Coord. Chem. Rev., 1994, 130, 275; ( $i$ ) R. Okazaki and R. West, Adv. Organomet. Chem., 1996, 39, 231; (j) T. Iwamoto, H. Sakurai and
M. Kira, Bull. Chem. Soc. Jpn., 1998, 71, 2741; (k) P. P. Power, J. Chem. Soc., Dalton Trans., 1998, 2939; (l) P. P. Power, Chem. Rev., 1999, 99, 3463; (m) M. Kira and T. Iwamoto, J. Organomet. Chem., 2000, 610, 236; (n) P. P. Gaspar and R. West, in The Chemistry of Organic Silicon Compounds, ed. Z. Rappoport and Y. Apeloig, Wiely, New York, 2nd edn, 1999, part 3, pp. 2463-2568; (o) M. Haaf, T. A. Schmedake and R. West, Acc. Chem. Res., 2000, 33, 704; (p) B. Gehrhus and M. F. Lappert, J. Organomet. Chem., 2001, 607-608, 209; $(q)$ N. J. Hill and R. West, J. Organomet. Chem., 2004, 689, 4165; (r) M. Kira, J. Organomet. Chem., 2004, 689, 4475; (s) O. Kuhl, Coord. Chem. Rev., 2004, 248, 411; $(t)$ R. W. Alder, M. E. Blake, L. Chaker, J. N. Harvey, F. Paolini and J. Schutz, Angew. Chem., Int. Ed., 2004, 43, 5896; (u) B. Gehrhus, P. B. Hitchcock, R. Pongtavornpinyo and L. Zhang, J. Chem. Soc., Dalton Trans., 2006, 15, 1847.

3 (a) M. J. Michalczyk, R. West and J. Michl, Organometallics, 1985, 4, 826; (b) B. D. Shephered, D. R. Powell and R. West, Organometallics, 1989, 8, 2664.

4 (a) M. Kira, T. Iwamoto and C. Kabuto, J. Am. Chem. Soc., 1996, 118, 10303; (b) T. Iwamoto, M. Tamura, C. Kabuto and M. Kira, Organometallics, 2003, 22, 2342; (c) T. Iwamoto and M. Kira, Chem. Lett., 1998, 277.

5 For instance, see: F. Bernardi, M. Olivucci and M. A. Robb, J. Photochem. Photobiol., A, 1997, 105, 365.

6 J. J. W. McDouall, K. Peasley and M. A. Robb, Chem. Phys. Lett., 1988, 148, 183.

7 For the sake of simplicity, the $\mathrm{SiH}_{3}$ substituent groups, instead of $t$ - $\mathrm{Bu}_{2} \mathrm{MeSi}$ groups, have been used in the present study. Of course, if one uses the above bulky group in the present theoretical study, the relative energies of CI and TS may change. Nevertheless, the basic concepts in these studies are still the same.

8 For reviews, see: (a) F. Bernardi, M. Olivucci and M. A. Robb, Isr. J. Chem., 1993, 265; (b) M. Klessinger, Angew. Chem., Int. Ed. Engl., 1995, 34, 549; (c) F. Bernardi, M. Olivucci and M. A. Robb, Chem. Soc. Rev., 1996, 321; (d) M. Klessinger, Pure Appl. Chem., 1997, 69, 773; (e) M. Klessinger and J. Michl, in Excited States and Photochemistry of Organic Molecules, VCH Publishers, New York, 1995.

9 M. J. Bearpark, M. A. Robb and H. B. Schlegel, Chem. Phys. Lett., 1994, 223, 269.

10 M. J. Frisch, G. W. Trucks, H. B. Schlegel, G. E. Scuseria, M. A. Robb, J. R. Cheeseman, G. Scalmani, V. Barone, B. Mennucci and G. A. Petersson, et al., Gaussian, Inc., Wallingford CT, 2013.

11 Recently, Matsuo, Tamao, and co-workers experimentally reported an interestingly substituted disilene molecule, (Z)1,2-di(1-prenyl)disilene, whose first excited state is theoretically estimated to be singlet. See: M. Kobayashi, N. Hayakawa, T. Matsuo, B. Li, T. Fukunaga, D. Hashizume, H. Fueno, K. Tanaka and K. Tamao, J. Am. Chem. Soc., 2016, 138, 758.

12 For discussion of excited singlet and triplet states of disilene, for instance, see a comprehensive study: 
G. A. Oyedepo and A. K. Wilson, J. Phys. Chem. A, 2010, 114, 8806.

13 The MO representation of silylene at the singlet excited state can be found in a recent work. See: M.-D. Su, Chem.-Eur. J., 2014, 20, 9419.

14 The fixed geometrical parameters in (A) Si-Rea and (B) C-Rea are given in ESI. $\dagger$

15 The reason that the Franck-Condon energy for Si-Rea-S $\left(67.8 \mathrm{kcal} \mathrm{mol}^{-1}\right)$ is much less than that for C-Rea-S $\mathbf{0}(116.8$ kcal $\mathrm{mol}^{-1}$ ), as already shown in Fig. 2 and 3 is easily understood with reference to the simple molecular systems, i.e., ethylene $\left(\mathrm{H}_{2} \mathrm{C}=\mathrm{CH}_{2}\right)$ and silene $\left(\mathrm{H}_{2} \mathrm{Si}=\mathrm{SiH}_{2}\right)$. The energy gap for the HOMO $(\pi)$ and the LUMO $\left(\pi^{*}\right)$ for ethylene is much larger than that for silene. Silene also has a higher HOMO $(\pi)$ and a lower LUMO $\left(\pi^{*}\right)$ than ethylene. For details, see ref. 2. 